\title{
Investigação de Etiologia Genética nas Demências Neurodegenerativas: Recomendações do Grupo de Neurogenética do Centro Hospitalar São João
}

\author{
Investigation of Genetic Etiology in Neurodegenerative \\ Dementias: Recommendations from the Centro Hospitalar \\ São João Neurogenetics Group
}

\author{
João MASSANO $\triangle^{1,2}$, Miguel LEÃO ${ }^{3,4}$, Carolina GARRETT ${ }^{1,5}$, em nome do Grupo de Neurogenética do Centro Hospitalar \\ São João
}

Colaboradores do artigo e membros do Grupo de Neurogenética do Centro Hospitalar São João:

Carla MOURA' ${ }^{3,6}$, Elsa AZEVEDO ${ }^{1,5}$, Joana GUIMARÃES ${ }^{1,5}$, João Paulo OLIVEIRA' ${ }^{3,7}$, Pedro CASTRO ${ }^{1,5}$

Acta Med Port 2016 Oct;29(10):675-679 - http://dx.doi.org/10.20344/amp.7583

\section{RESUMO}

Nos últimos anos foram identificadas várias mutações genéticas causadoras das demências neurodegenerativas mais frequentes (doença de Alzheimer e demência fronto-temporal). Estes avanços, em conjunto com a complexidade das relações entre genótipo e fenótipo, e os próprios custos associados ao processo de diagnóstico genético, tornaram por vezes difícil aos clínicos a escolha de um plano racional para a investigação da etiologia genética das demências neurodegenerativas. O Grupo de Neurogenética do Centro Hospitalar, grupo multidisciplinar de Neurologistas e Geneticistas com interesse especial na área das doenças neurogenéticas delineou recomendações de consenso para a investigação da etiologia genética da doença de Alzheimer e demência fronto-temporal na prática clínica, tendo por base documentos de consenso internacionais (contendo atualmente informação parcialmente desatualizada) e a evidência científica publicada sobre este tópico. A doença de Alzheimer pode ser causada por mutações nos genes PSEN1, $P S E N 2$ e APP. Não é recomendada a genotipagem da APOE para o diagnóstico ou aconselhamento genético na doença de Alzheimer. A demência fronto-temporal pode ser causada por mutações em vários genes como c9orf72, PGRN, MAPT, TBK1, VCP, SQSTM1 e UBQLN2. Este documento aborda de forma pragmática o processo utilizado para o diagnóstico genético da doença de Alzheimer e demência fronto-temporal, com recomendações específicas para ambas as situações.

Palavras-chave: Demência/diagnóstico; Demência Frontotemporal/diagnóstico; Demência Frontotemporal/genética; Demência/genética; Doença de Alzheimer/diagnóstico; Doença de Alzheimer/genética.

\section{ABSTRACT}

In the past few years several gene mutations have been identified as causative of the most frequent neurodegenerative dementias (Alzheimer disease and frontotemporal dementia). These advances, along with the complex phenotype-genotype relationships and the costs associated with genetic testing, have often made it difficult for clinicians to decide with regard to a rational plan for the investigation of the genetic etiology of the degenerative dementias. The Centro Hospitalar São João Neurogenetics Group, a multidisciplinary team of Neurologists and Geneticists with special interest in neurogenetic disorders, devised consensus recommendations for the investigation of the genetic etiology of Alzheimer disease and frontotemporal dementia in clinical practice, based on international consensus documents (currently containing partly outdated information) and published scientific evidence on this topic. Alzheimer disease may be caused by mutations in PSEN1, PSEN2 and APP. APOE genotyping is not recommended for the diagnostic or genetic counseling purposes in Alzheimer disease. Frontotemporal dementia may be caused by mutations in several genes such as c9orf72, PGRN, MAPT, TBK1, VCP, SQSTM1, and UBQLN2. This paper pragmatically approaches the process of genetic diagnosis in Alzheimer disease and frontotemporal dementia, with specific recommendations for both disorders.

Keywords: Alzheimer Disease/diagnosis; Alzheimer Disease/genetics; Dementia/diagnosis; Dementia/genetics; Frontotemporal Dementia/diagnosis; Frontotemporal Dementia/genetics; Portugal.

\section{INTRODUÇÃO}

A designação 'demência' refere-se a um quadro clínico em que existe declínio cognitivo a partir de um nível de funcionamento prévio, determinando uma diminuição da capacidade de realização das atividades de vida diária por parte do indivíduo afetado. O termo 'demência' é uma designa-

ção sindrómica e não tem implicações etiológicas, existindo inúmeras causas para esta situação. As demências podem ser essencialmente primárias (neurodegenerativas) ou secundárias (aqui incluem-se, por exemplo, a demência pós-traumática ou pós-infeciosa, a hidrocefalia de pressão

\footnotetext{
1. Departamento de Neurociências Clínicas e Saúde Mental. Faculdade de Medicina. Universidade do Porto. Porto. Portugal.

2. Serviço de Neurologia. Hospital Pedro Hispano. Unidade Local de Saúde de Matosinhos. Matosinhos. Portugal.

3. Serviço de Genética. Faculdade de Medicina. Universidade do Porto. Porto. Portugal.

4. Unidade de Neuropediatria. Serviço de Pediatria. Centro Hospitalar São João. Porto. Portugal.

5. Serviço de Neurologia. Centro Hospitalar São João. Porto. Portugal.

6. Serviço de Otorrinolaringologia. Centro Hospitalar São João. Porto. Portugal.

7. Serviço de Nefrologia. Centro Hospitalar São João. Porto. Portugal.

$\triangle$ Autor correspondente: João Massano. jmassano@med.up.pt

Recebido: 06 de março de 2016 - Aceite: 02 de setembro de 2016 | Copyright @ Ordem dos Médicos 2016
} 
normal, a demência devida a défices vitamínicos ou alterações tiroideias e o défice cognitivo/demência vascular). ${ }^{1-3}$

Uma proporção significativa de demências neurodegenerativas é determinada por alterações genéticas de transmissão autossómica dominante, sobretudo no caso da demência fronto-temporal $(20 \%-50 \%)$ e muito menos frequentemente a doença de Alzheimer (provavelmente $<1 \%$ ), números que variam consoante as séries e as populações estudadas..$^{1,4}$ Estas situações observam-se com frequência na prática clínica diária, sendo muitas vezes considerada a pertinência da realização de testes moleculares. Uma das questões que os familiares mais colocam na consulta de Neurologia é precisamente a possibilidade de a situação clínica em causa ser hereditária ou não. $\mathrm{Na}$ maioria das situações é possível depreender para cada caso se este é esporádico ou hereditário, mas nem sempre a resposta é linear. Por outro lado, o surgimento de sintomas em idade relativamente jovem ou a existência de uma história familiar positiva podem constituir motivos para colocar a questão do diagnóstico genético e do aconselhamento apropriado neste âmbito.

Este documento apresenta um protocolo de estudo genético para as formas hereditárias das demências neurodegenerativas (doença de Alzheimer e demência fronto-temporal) para utilização na prática clínica, proposto pelo Grupo de Neurogenética (GNgen) do Centro Hospitalar São João (CHSJ).

\section{METODOLOGIA PARA ELABORAÇÃO DO PROTOCO- LO}

O GNgen do CHSJ, formalizado em 2011 com a criação da Consulta de Grupo, reúne periodicamente para promover a partilha de conhecimento e a discussão clínica e científica no âmbito das doenças neurogenéticas. É constituído por um conjunto definido de profissionais provenientes de diferentes áreas técnico-científicas (lista de membros no final do artigo). As reuniões são abertas a todos os médicos do CHSJ que nelas queiram participar, nomeadamente os que têm interesse particular nestas doenças ou na Genética Médica, e médicos em formação.

A elaboração do protocolo de estudo genético nas demências neurodegenerativas foi entregue a um núcleo de autores que estabeleceram a metodologia de trabalho, pesquisaram e analisaram a literatura, coligiram os dados disponíveis e elaboraram uma primeira versão do protocolo, que foi enviada a todos os membros do GNgen por correio eletrónico. As várias sugestões obtidas por via electrónica foram debatidas e incorporadas na versão definitiva que foi apresentada, debatida e aprovada por consenso em reunião do GNgen.

\section{ESTUDO GENÉTICO NAS DEMÊNCIAS NEURODEGE- NERATIVAS: RECOMENDAÇÕES CLÍNICAS}

Este protocolo foi elaborado com base nas linhas de orientação emitidas conjuntamente pela European Federation of Neurological Societies (EFNS) e pela European Neurological Society (ENS), publicadas em 2012 no European
Journal of Neurology. ${ }^{5}$ Estas recomendações são consideravelmente vagas, pelo que foram mantidas neste protocolo as recomendações prévias provenientes da EFNS, ${ }^{6,7}$ dado que não são mutuamente exclusivas. Os níveis de evidência apontados para cada recomendação são os utilizados de forma padronizada a nível internacional; sempre que não existam dados de Medicina Baseada na Evidência os peritos envolvidos na elaboração destas linhas de orientação emitiram uma recomendação consensual baseada na sua própria experiência e conhecimentos ('boa prática clínica'); os artigos originais estão facilmente acessíveis na world wide web, para a consulta de mais detalhes. A esta base de trabalho foram acrescentadas alterações adicionais relevantes, decorrentes da evolução do conhecimento científico entretanto ocorrida desde a publicação dos documentos de referência mencionados. ${ }^{8-11}$

Na maioria das situações, o diagnóstico genético molecular será realizado em doentes com história familiar claramente consistente com uma forma monogénica da doença (em regra com padrão de hereditariedade autossómica dominante) ou em casos esporádicos em que se verifiquem circunstâncias especiais (e.g. idade de início dos sintomas invulgarmente baixa). Em todo o caso há um número considerável de casos aparentemente esporádicos em que são detetáveis mutações patogénicas e pode ser lícito também proceder ao estudo genético nestas situações, dependendo das circunstâncias clínicas e da vontade dos envolvidos, após o devido esclarecimento detalhado. Por outro lado, pode ser necessário considerar a pesquisa de mutações em genes associados de forma pouco frequente ao fenótipo em causa (Tabela 1).

As recomendações relativas ao estudo molecular genético na doença de Alzheimer (DA) e na demência fronto-temporal (DFT) são as seguintes:

1. Perante um diagnóstico clínico de DA, a pesquisa de mutações é útil para o aconselhamento genético nos casos de transmissão autossómica dominante de início precoce (abaixo dos 65 anos). Os genes devem ser testados pela ordem decrescente de probabilidade de encontrar mutações, o que implica o seguinte estudo sequencial: PSEN1, APP e finalmente PSEN2 (nível B de evidência, tal como definido no documento original da $\mathrm{EFNS}^{6}$ ). O rastreio genético pode ser ponderado nos casos aparentemente esporádicos de início precoce (boa prática clínica). Tendo em conta a relação custo-benefício pode ser considerada a realização de estudos moleculares em painéis multigénicos.

2. O alelo $A p o E \varepsilon 4$ é um importante factor de risco genético para DA, mas não é necessário nem suficiente para o aparecimento da mesma. Não existe evidência suficiente relativamente à utilidade clínica da genotipagem $A P O E$, pelo que não é recomendada a sua realização (recomendação do GNgen do CHSJ).

3. Se o diagnóstico clínico for de síndrome de DFT autossómica dominante, a realização de testes moleculares para a pesquisa de mutações está claramente indicada, sendo útil para aconselhamento genético (nível $B$ de 
Tabela 1 - Demências neurodegenerativas hereditárias

Situação patológica

Síndromes de demência fronto-temporal (DFT)

(a designação neuropatológica é degenerescência lobar fronto-temporal, DLFT)

Comentários:
Características clínicas mais típicas, associações génicas e neuropatológicas

Características clínicas mais típicas, associações génicas e neuropatológicas

A causa mais comum de demência neurodegenerativa no mundo. A idade típica de início da forma esporádica é acima dos 65 anos. A maioria dos casos hereditários ocorre abaixo dos 60 anos de idade (alguns na terceira década de vida) e a transmissão é autossómica dominante (apenas uma família relatada com transmissão autossómica recessiva, com mutação no gene $A P P$ ). $O$ fenótipo cognitivo típico é dominado desde cedo por défices de memória episódica (sobretudo para factos recentes), a que se associam outros défices corticais (agnosia, afasia, apraxia, disfunção executiva e visuo-espacial). Progressão geralmente lenta na forma esporádica, ao longo de uma década ou mais, mas mais rápida nas hereditárias. Há fenótipos que incluem paraparésia espástica, associados a mutações do gene PSEN1.

Globalmente menos frequente que a DA, tende a ocorrer em faixas etárias mais jovens (45 - 60 anos) e com muito maior probabilidade de ter causa monogénica. Há três fenótipos principais: (1) variante comportamental (ou simplesmente demência fronto-temporal), caracterizada por alterações de comportamento social e de personalidade, perda de empatia, disfunção executiva, comportamentos repetitivos e alteração bizarra das preferências alimentares e défices de linguagem; (2) afasia progressiva não fluente, que cursa com uma afasia de tipo motor, com perda do gramatismo, parafasias, compreensão mantida até fases tardias, agravando progressivamente até ao mutismo completo; (3) demência semântica, caracterizada por uma perda progressiva do conhecimento de factos e conceitos e as relações entre estes, surgindo mais tarde agnosia visual e alteração de toda a compreensão linguística.

Há vários subtipos de DLFT, de acordo com as alterações neuropatológicas e genéticas subjacentes:

- DLFT-tau (histopatologia com deposição de proteína tau): fenótipo de variante comportamental de DFT, com ou sem parkinsonismo (FTDP-17); ou afasia progressiva não fluente; casos raros associados a fenótipo semelhante a Doença de Alzheimer.

- DLFT-TDP43 (histopatologia com deposição de TDP43): fenótipo de variante comportamental de DFT, com ou sem parkinsonismo, com ou sem doença de neurónio motor; ou demência semântica; ou afasia progressiva não fluente; casos raros associados aos fenótipos de síndrome córtico-basal e paralisia supranuclear progressiva. A coexistência de DFT com doença de neurónio motor (DNM) e/ou psicose no caso índice, ou existência de história familiar de qualquer destas alterações, é muito sugestiva de mutação no C9ORF72. Esta é a alteração genética mais frequente na DFT familiar e esporádica, e é a causa mais frequente de DFT-DNM. As mutações no gene TBK1 são a segunda causa mais frequente de DFT-DNM, havendo casos com DFT isolada ou DNM isolada; raramente o fenótipo é de afasia não fluente progressiva. Estes doentes têm défice de memória e desorientação (tempo e/ou espaço) cedo no curso da doença e a idade de início pode ser tardia (até à oitava década de vida, média 63 anos). Os sintomas de parkinsonismo parecem ser mais frequentes do que nos casos com mutação C9ORF72.

- DLFT-FUS* (histopatologia com deposição de proteína FUS): fenótipo de variante comportamental de DFT, com ou sem parkinsonismo, tipicamente em idade muito jovem ( $<40$ anos); maioria dos casos sem mutação FUS. Atrofia do núcleo caudado é frequentemente observada na imagem cerebral estrutural.

- DFT ligada ao cromossoma 3*: fenótipo de variante comportamental de DFT.

- DFT associada a mutações do VCP*: variante comportamental de DFT, com ou sem miosite de corpos de inclusão e com ou sem doença de Paget óssea. Raramente fenótipo de variante comportamental de DFT com doença de neurónio motor.

- DFT associada a mutações do UBQLN2*: fenótipo de variante comportamental de DFT com doença de neurónio motor, de transmissão ligada ao X.

- DFT associada a mutações do SQSTM1*: fenótipo de variante comportamental de DFT com ou sem doença de neurónio motor, com ou sem Doença de Paget óssea.

- DFT associada a mutações do TARDBP*: fenótipo de variante comportamental de DFT com doença de neurónio motor.

- DFT familiar de tipo britânico* e tipo dinamarquês*: início dos sintomas entre os 20 e os 50 anos; espasticidade e ataxia precocemente no tipo britânico; cataratas, ataxia e surdez precocemente no tipo dinamarquês.
Locus, gene

14q24.3, PSEN1

21q21, $A P P$

1q31-q42, PSEN2

(há casos raros de fenótipo de DFT associados a mutações de PSEN1 e PSEN2)

17q21.1, MAPT

C9ORF72

7q21.32, PGRN

12q14.2, TBK1

16p11.2, FUS

3p11.2, CHMP2B

9p13-912, VCP

Xp11.21, UBQLN2

5q35.3, SQSTM1

1p36.22, TARDBP

13q14.3, BR/2

O asterisco $\left(^{*}\right)$ assinala as formas monogénicas raras de DFT. PSEN1: presenilin-1; PSEN2: presenilin-2; APP: amyloid precursor protein; MAPT: microtubule associated protein tau; PGRN: progranulin; C9ORF72: chromosome 9 open reading frame 72; TBK1: TANK-binding protein kinase 1; FUS: fused in sarcoma; CHMP2B: charged multivesicular body protein 2B; VCP: valosin containing protein; BRI2=ITM2B: integral membrane protein 2B; UBQLN2: ubiquilin 2; SQSTM1: sequestosome 1; TARDBP: TAR DNA-binding protein 
evidência ${ }^{6}$ ). Pode também ser ponderada a realização de testes de diagnóstico molecular genético em casos aparentemente esporádicos (boa prática clínica), tendo em conta que são encontradas mutações em cerca de $5 \%-10 \%$ destes doentes.

4. A alteração genética mais frequente nos casos de DFT é a expansão patológica do número de repetições do hexanucleótido $\mathrm{G}_{4} \mathrm{C}_{2}$ em $C 90 R F 72$, pelo que deve ser o primeiro teste a realizar na ausência de alterações fenotípicas que aconselhem outra escolha. Deve ser igualmente o primeiro teste a realizar quando no caso índice coexiste DFT e doença do neurónio motor (DNM) e/ou psicose, ou caso haja história familiar de qualquer uma destas situações (recomendação do GNgen do CHSJ).

5. Se a pesquisa da expansão patológica em C9ORF72 for negativa deve prosseguir-se para a pesquisa de mutações nos genes PGRN, TBK1 e MAPT. Os artigos de Paulos e Massano ${ }^{4}$ e LeBer et a/ ${ }^{9}$ apresentam exemplos de algoritmos de decisão que podem ser utilizados na prática clínica (recomendação do GNgen do CHSJ).

6. Se o fenótipo observado for de DFT com DNM (ou se houver casos de DNM na família do caso-índice) e não houver mutação patológica do C9ORF72, devem pesquisar-se de seguida mutações do gene TBK1 e, se ausentes, do gene SQSTM1 (recomendação do GNgen do CHSJ).

7. Nos raros casos de DFT com história familiar sugestiva de transmissão ligada ao cromossoma $X$ devem ser pesquisadas mutações no gene UBQLN2 em primeiro lugar (recomendação do GNgen do CHSJ).

8. A realização de testes genéticos depende da verificação do cumprimento das recomendações constantes dos pontos anteriores e obedece às disposições legais em vigor, designadamente a Lei $n^{\circ} 12 / 2005$ de 26 de Janeiro e o Decreto-Lei n. ${ }^{\circ} 131 / 2014$, de 29 de Agosto.

9. Este protocolo será revisto sempre que seja entendido necessário e oportuno.

Existem ainda outras formas de demência de causa monogénica, a maioria das quais não está contemplada em quaisquer linhas de orientação ou documentos de consenso internacionais para diagnóstico genético de demência na prática clínica. São situações raras e algumas claramente típicas de populações com origem étnico-geográfica específica. Na eventualidade de ser julgado necessário testar alguma destas formas específicas recomenda-se a discussão do caso clínico concreto em ambiente multidisciplinar especializado antes de se prosseguir eventualmente para o teste laboratorial (prática habitual do GNgen do CHSJ).

\section{CONSIDERAÇÕES FINAIS}

Os avanços tecnológicos e laboratoriais, em especial o surgimento das técnicas de sequenciação de nova gera- ção, como o whole exome sequencing, têm permitido pesquisar mutações em vários genes simultaneamente. Tem havido esforços consideráveis no sentido de disponibilizar comercialmente 'painéis' de teste para variadas doenças neurológicas, o que já sucede atualmente para a DA e a DFT, por exemplo. Será sensato monitorizar a evolução da evidência relativamente a estas técnicas, pois pode tornar-se economicamente mais viável e útil do ponto de vista clínico recorrer a esta metodologia no futuro, em detrimento da atual estratégia de pesquisa sequencial dos vários genes potencialmente envolvidos na DA e na DFT.

Atualmente não é reconhecida a utilidade da biópsia cerebral no diagnóstico e orientação terapêutica da generalidade dos doentes com demência neurodegenerativa, devido aos riscos implicados e ao facto dos respectivos resultados não contribuírem para modificar a estratégia terapêutica. ${ }^{12} \mathrm{O}$ facto de não ser analisado todo o cérebro limita a acuidade diagnóstica e as correlações entre a genética e a neuropatologia são atualmente limitadas, pelo que não é lícito fazer biópsia cerebral com o intuito de guiar a escolha do teste genético de diagnóstico. Poderá, no entanto, ser ponderado o recurso a biópsia cerebral com intuito diagnóstico em situações particulares, nomeadamente em caso de necessidade de exclusão de diagnósticos diferenciais com potencial tratamento específico, cuja definição está fora do âmbito destas normas de orientação.

A escolha do teste molecular genético para o diagnóstico das demências degenerativas é complexo, dadas as especificidades clínicas e a baixa frequência de algumas das situações aqui referidas. Assim, esta escolha deve ser feita mediante observação especializada prévia por profissionais com elevada diferenciação nesta área.

\section{PROTECÇÃO DE PESSOAS E ANIMAIS}

Os autores declaram que os procedimentos seguidos estavam de acordo com os regulamentos estabelecidos pelos responsáveis da Comissão de Investigação Clínica e Ética e de acordo com a Declaração de Helsínquia da Associação Médica Mundial.

\section{CONFIDENCIALIDADE DOS DADOS}

Os autores declaram ter seguido os protocolos do seu centro de trabalho acerca da publicação de dados.

\section{CONFLITOS DE INTERESSE}

Os autores declaram não terem qualquer conflito de interesse relativamente ao presente artigo.

\section{FONTES DE FINANCIAMENTO}

Este trabalho não foi subsidiado por quaisquer fundos provenientes de entidades no domínio público ou privado.

\section{REFERÊNCIAS}

1. Real R, Massano J. Demências. In: Sá MJ, editor. Neurologia Clínica - Compreender as Doenças Neurológicas, $2^{a}$ ed. Porto: Edições Fernando Pessoa; 2014. p. 475-506.

2. Scott KR, Barrett AM. Dementia syndromes: evaluation and treatment. Expert Rev Neurother. 2007;7:407-22.

3. Feldman HH, Jacova C, Robillard A, Garcia A, Chow T, Borrie M, 
et al. Diagnosis and treatment of dementia: 2. Diagnosis. CMAJ. 2008;178:825-36.

4. Paulos JP, Massano J. Clinical, genetic and neuropathological features of frontotemporal dementia: an update and guide. Acta Med Port. 2013;26:392-401.

5. Sorbi S, Hort J, Erkinjuntti T, Fladby T, Gainotti G, Gurvit H, et al. EFNS-ENS Guidelines on the diagnosis and management of disorders associated with dementia. Eur J Neurol. 2012;19:1159-79.

6. Burgunder JM, Finsterer J, Szolnoki Z, Fontaine B, Baets J, Van Broeckhoven C, et al. EFNS guidelines on the molecular diagnosis of channelopathies, epilepsies, migraine, stroke, and dementias. Eur J Neurol. 2010;17:641-8

7. Hort J, O'Brien JT, Gainotti G, Pirttila T, Popescu BO, Rektorova I, et al; on Behalf of the EFNS Scientist Panel on Dementia. EFNS guidelines for the diagnosis and management of Alzheimer's disease. Eur J Neurol. 2010;17:1236-48.

8. Van der Zee J, Gijselinck I, Dillen L, Van Langenhove T, Theuns J, Engelborghs S, et al. European Early-Onset Dementia Consortium.
A pan-European study of the C9orf72 repeat associated with FTLD: geographic prevalence, genomic instability, and intermediate repeats. Hum Mutat. 2013;34:363-73.

9. Le Ber I, Camuzat A, Guillot-Noel L, Hannequin D, Lacomblez L, Golfier $V$, et al. C9ORF72 repeat expansions in the frontotemporal dementias spectrum of diseases: a flow-chart for genetic testing. J Alzheimers Dis. 2013;34:485-99.

10. Gijselinck I, Van Mossevelde S, van der Zee J, Sieben A, Philtjens S, Heeman B, et al. Loss of TBK1 is a frequent cause of frontotemporal dementia in a Belgian cohort. Neurology. 2015;85:2116-25.

11. Van Mossevelde S, van der Zee J, Gijselinck I, Engelborghs S, Sieben A, Van Langenhove $T$, et al. Belgian Neurology consortium. Clinical features of TBK1 carriers compared with C9orf72, GRN and nonmutation carriers in a Belgian cohort. Brain. 2016;139:452-67.

12. Schott JM, Reiniger L, Thom M, Holton JL, Grieve J, Brandner S, et al. Brain biopsy in dementia: clinical indications and diagnostic approach. Acta Neuropathol. 2010;120:327-41. 